\title{
SARS-CoV-2 antibodies in Ontario health care workers during and after the first wave of the pandemic: a cohort study
}

\author{
Michelle Science MD MSc, Shelly Bolotin PhD, Michael Silverman MD, Jeya Nadarajah MD MSc, \\ Bryan Maguire MSc, Rulan S. Parekh MD MS, Allison McGeer MD MSc, Kevin L. Schwartz MD MSc, \\ Laura Alexander MHSc, Upton Allen MD MBBS, Archchun Ariyarajah MSc, Lucas Castellani MD MBBS, \\ Ronald D. Cohn MD, Mark Downing MD, Kevin Katz MD, Kescha Kazmi MD, Jerome A. Leis MD MSc, \\ Derek Liu RN, Jeffrey M. Pernica MD MSc, Jane E. Schneiderman PhD, Maya Sumaida RN, \\ Aaron Campigotto MD
}

\section{Abstract}

Background: Health care workers have a critical role in the pandemic response to COVID-19 and may be at increased risk of infection. The objective of this study was to assess the seroprevalence of SARS-CoV-2 immunoglobulin G (IgG) antibodies among health care workers during and after the first wave of the pandemic.

Methods: We conducted a prospective multicentre cohort study involving health care workers in Ontario, Canada, to detect IgG antibodies against SARS-CoV-2. Blood samples and self-reported questionnaires were obtained at enrolment, at 6 weeks and at 12 weeks. A community hospital, tertiary care pediatric hospital and a combined adult-pediatric academic health centre enrolled participants from Apr. 1 to Nov. 13, 2020. Predictors of seropositivity were evaluated using a multivariable logistic regression, adjusted for clustering by hospital site.

Results: Among the 1062 health care workers participating, the median age was 40 years, and 834 (78.5\%) were female. Overall, $57(5.4 \%)$ were seropositive at any time point $(2.5 \%$ when participants with prior infection confirmed by polymerase chain reaction testing were excluded). Seroprevalence was higher among those who had a known unprotected exposure to a patient with COVID-19 $(p<0.001)$ and those who had been contacted by public health because of a nonhospital exposure $(p=0.003)$. Providing direct care to patients with COVID-19 or working on a unit with a COVID-19 outbreak was not associated with higher seroprevalence. In multivariable logistic regression, presence of symptomatic contacts in the household was the strongest predictor of seropositivity (adjusted odds ratio $7.15,95 \%$ confidence interval 5.42-9.41).

Interpretation: Health care workers exposed to household risk factors were more likely to be seropositive than those not exposed, highlighting the need to emphasize the importance of public health measures both inside and outside of the hospital.

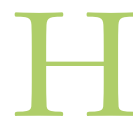
ealth care workers have a critical role in the pandemic response to COVID-19, potentially increasing their risk for infection as a consequence..$^{1-3} \mathrm{It}$ is important to understand risk factors that may predispose health care workers to SARS-CoV-2 infection and guide targeted interventions and improved direct health and safety measures. Understanding risk and effective preventive measures is important to both ensure a healthy essential workforce and protect patients and health care workers from potential nosocomial transmission.

Estimates of SARS-CoV-2 infection using only molecular diagnostic tests can lead to substantial testing bias and may underestimate the prevalence of infection. ${ }^{4}$ In contrast to molecular tests, which primarily detect acute infection, serologic testing can assist in assessing prior infection and identifying cases that may not have had acute diagnostic testing. As such, the use of serologic assays targeting SARS-CoV-2

Competing interests: See the end of the article.

This article has been peer reviewed.

Correspondence to: Michelle Science, michelle.science @ sickkids.ca; Aaron Campigotto, aaron.campigotto@ sickkids.ca

CMAJ Open 2021 October 12. DOI:10.9778/cmajo.20210044 
antibodies is a useful tool to understand the epidemiology of COVID-19 within a population and the burden of previous mild or asymptomatic infection. ${ }^{5}$ Serology tests typically have a high sensitivity for previous SARS-CoV-2 infection when testing occurs more than 14 days after the onset of symptoms. ${ }^{6,7}$

Some studies assessing whether SARS-CoV-2 seropositivity in health care workers is elevated compared with the general population have reported higher seroprevalence. ${ }^{8-10} \mathrm{In}$ addition to risk factors shared with the general population, such as age, ethnicity, household exposure to SARS-CoV-2 and burden of COVID-19 in the residing communities, there are potential risk factors specific to the hospital, including general inpatient care, direct care of patients with COVID-19 and working on a COVID-19 ward. $^{8,11-15}$ It is therefore critical to place the risk of health care workers acquiring COVID19 in a local clinical context, which addresses hospital safety practices and also community disease prevalence.

The purpose of this study was to assess the overall seroprevalence of SARS-CoV-2 immunoglobulin G (IgG) antibodies in a population of health care workers within Ontario during and immediately after the first wave of the pandemic, and to explore factors associated with seropositivity. We also sought to explore the durability of antibodies specific to SARS-CoV-2 over time.

\section{Methods}

\section{Study design}

We conducted a prospective multicentre cohort study involving health care workers in Ontario, Canada, to detect IgG antibodies against SARS-CoV-2. The study was proposed to hospitals across Ontario through an infection prevention and control community of practice with representation from more than 30 hospitals. After review and approval of the protocol, interested sites obtained research ethics and legal approvals, leading to variable start dates. The sites that completed recruitment during and immediately after the first wave (Apr. 1 to Nov. 13, 2020) were included in this analysis.

\section{Setting}

Three hospitals from 3 Ontario regions ${ }^{16}$ participated during the study period: The Hospital for Sick Children (SickKids), a tertiary care pediatric hospital in Toronto, Ontario (Toronto Region); London Health Sciences Centre, an academic centre in London, Ontario, consisting of 2 hospitals including a combined pediatric-adult hospital (South West Region); and Markham Stouffville Hospital, a community hospital in Markham, Ontario (Central East Region). Infection prevention and control guidelines were the same across the hospitals and aligned with provincial guidelines, including use of droplet and contact precautions for routine care of patients with suspected or confirmed COVID-19, with N95 respirators used for aerosol-generating medical procedures. ${ }^{17}$ Information on the number of patients with COVID-19 receiving treatment during the study period was collected from each hospital.

\section{Participants}

Health care workers invited to participate included health care professionals, defined as physicians, nurses and nurse practitioners; allied health workers, defined as phlebotomists, respiratory therapists, social workers, dieticians, diagnostic imaging staff, physiotherapists, occupational therapists and dentistry staff; and auxiliary health care workers (as defined by the World Health Organization as workers who may have had contact with patients, their body fluids or their environments ${ }^{18}$ ), including environmental services, patient transport and laboratory personnel, and ward clerical workers.

Recruitment tools included posters, all-staff emails from leadership, computer screen savers and a website (http:// cancovid19plasma.ca/healthcare-worker-serology/) that provided general information about the study and contact information for the study coordinators. In addition, we specifically recruited health care workers who worked in emergency departments, COVID-19 wards or units and intensive care units, and those involved with aerosol-generating medical procedures (e.g., anesthesia and respiratory therapy) through directed communication at departmental meetings or emails by clinical directors, as these groups may have had a higher risk of exposure to SARS-CoV-2.

\section{Procedures}

Blood samples and self-reported questionnaires were obtained from all enrolled participants at baseline (i.e., enrolment), at 6 weeks and at 12 weeks. Blood samples were separated by centrifugation, and serum was stored frozen at $-80^{\circ} \mathrm{C}$. Questionnaires asked about potential risk factors for SARS-CoV-2 exposure and mitigation strategies, including travel history, care of patients with COVID-19, known exposure (occupational or otherwise) to a confirmed case of COVID-19, perceived adherence to physical distancing measures and the type of personal protective equipment (PPE) used during patient encounters (all patients and patients with suspected or confirmed COVID-19) (Appendix 1, available at www.cmajopen. ca/content/9/4/E929/suppl/DC1). In addition, all participants were emailed weekly to request that they report any new symptoms.

Our proposed sample size of at least 1000 health care workers would allow us to determine seropositivity at baseline with an $80 \%$ probability that the confidence interval (CI) has a precision of $\pm 1.5 \%$, assuming a seroprevalence of $5 \%$ (80\% power, $\alpha$ of 0.05 ).

\section{Outcome}

The EUROIMMUN Anti-SARS-CoV-2 IgG enzyme-linked immunoassay (ELISA) ${ }^{19}$ was used for testing in accordance with the manufacturer's directions on the EUROIMMUN Analyzer I. This Health Canada-approved semiquantitative assay detects a recombinant S1 protein of SARS-CoV-2. Interpretation was based on the index values (signal to cut-off ratios) of less than 0.8 reported as negative, 0.8 or greater to less than 1.1 as borderline, and 1.1 or greater as positive. ${ }^{19}$ This assay has a reported sensitivity of greater than $90 \%$ and specificity of greater than $98 \%$ in patients 15 days or more 
post-symptom onset. ${ }^{20}$ All testing was performed at the Microbiology Laboratory at SickKids.

\section{Statistical analysis}

We reported continuous variables using the mean and standard deviation or median and interquartile range as appropriate. We reported numbers and percentages for dichotomous outcomes. Proportion of samples seropositive at each time point (baseline, 6 weeks and 12 weeks) was calculated overall and stratified by whether participants had a known SARSCoV-2 infection before enrolment. The proportions with seropositive results at each time point were compared between sites using $\chi^{2}$ tests. Spaghetti plots were used to display antibody responses over time.

Detailed information on several potential predictors will be studied in a larger longitudinal study that is ongoing. Given the small number of seropositive participants, we focused this analysis on potential hospital risk factors and household exposure, and included only 5 predictors in the multivariable model using the 10 events per variable rule of thumb. We targeted the univariable analyses to hospital risk factors (working on a unit with a COVID-19 outbreak, providing care for patients with COVID-19, having had an unprotected COVID-19 exposure) and nonhospital risk factors (symptomatic household contacts as defined by participant, contacted by public health about exposure) and evaluated the relation with seropositivity using the $\chi^{2}$ or Fisher exact test. All analyses were based on the baseline questionnaire responses. The multivariable logistic regression model included predictors identified a priori including age, sex, race or ancestry, a nonhospital risk factor (symptomatic contacts in the household) and a hospital risk factor (care of patients with COVID-19). We used generalized estimating equations with an exchangeable correlation structure to adjust for clustering at the site. A sensitivity analysis was conducted removing patients with known infection at baseline.

All estimates are presented with 95\% CIs. A $p$ value less than 0.05 was considered significant. All analyses were conducted using R (R Core Team, 2020).

\section{Ethics approval}

Research ethics approval was obtained by the Clinical Trials Ontario Research Ethics Board (Project ID 3182), with local site approvals as required. All participants provided informed consent.

\section{Results}

This analysis includes data from the first 3 hospitals recruited to participate in our study. A total of 2065 health care workers contacted the study team to learn more about the study, and 1082 consented to participate. Of those who consented, 1062 health care workers had baseline information available and bloodwork completed, and were included in the study from SickKids $(n=376)$, London Health Sciences Centre $(n=349)$ and Markham Stouffville Hospital $(n=337)$. This resulted in a total of 1062 baseline tests, 1042 six-week samples and
966 twelve-week samples (Figure 1). Over the study period, each hospital saw more than 100 patients with COVID-19. The range of timing of recruitment and sample collection at each site is shown in Figure 2.

The median age of health care workers was 40 (interquartile range $32-51)$ years, and $834(78.5 \%)$ were female (Table 1). Participants were predominantly nurses from inpatient units, critical care and the emergency department. Most participants racially self-identified as White, followed by Asian, with less than $2 \%$ self-identifying for each of Black, Hispanic, and Inuit, First Nations and Métis.

\section{Overall seropositivity}

Overall, 57/1062 (5.4\%) of health care workers were seropositive at any time point, of which 31 (54.4\%) had a history of confirmed SARS-CoV-2 infection by polymerase chain reaction (PCR) testing before enrolment. An additional 9 participants had previous confirmed SARS-CoV-2 infection but were seronegative. Of the 1022 health care workers with no confirmed SARS-CoV-2 infection before enrolment (i.e., excluding those with known recruitment bias), 26 (2.5\%) were seropositive at any time point over the study (Table 2). Seroprevalence varied minimally by time point (Figure 2 ), and there was no significant difference in seroprevalence by site $(p=0.1)$.

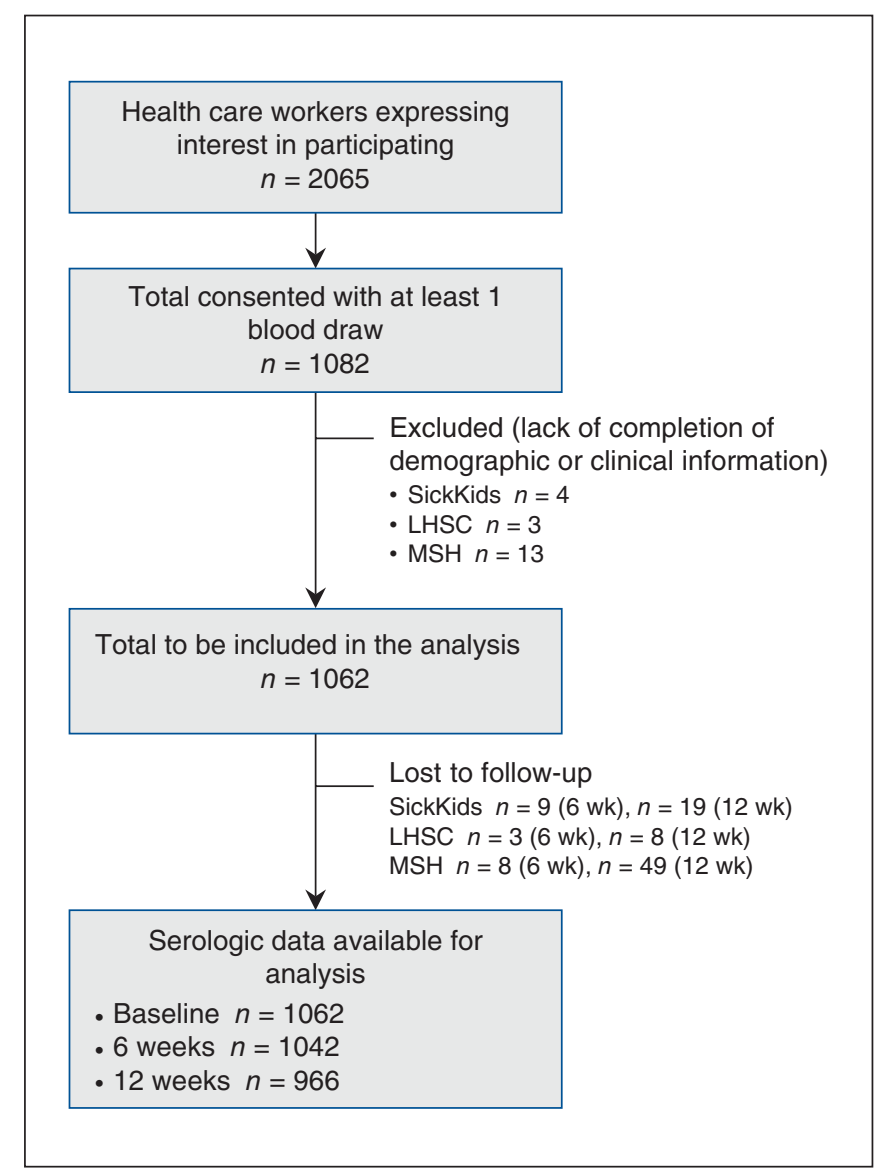

Figure 1: Participant inclusion flow diagram. Note: $L H S C=$ London Health Sciences Centre, MSH = Markham Stouffville Hospital, SickKids $=$ The Hospital for Sick Children. 


\section{Research}

\section{SickKids}
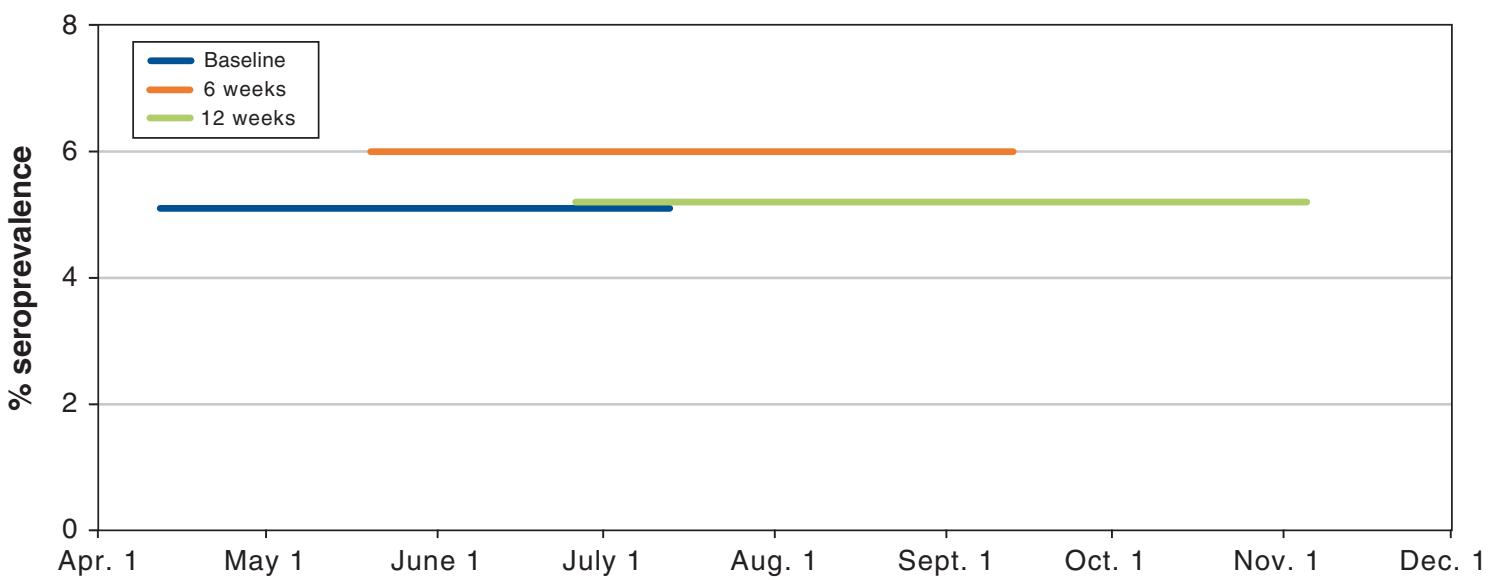

Date, 2020

\section{LHSC}

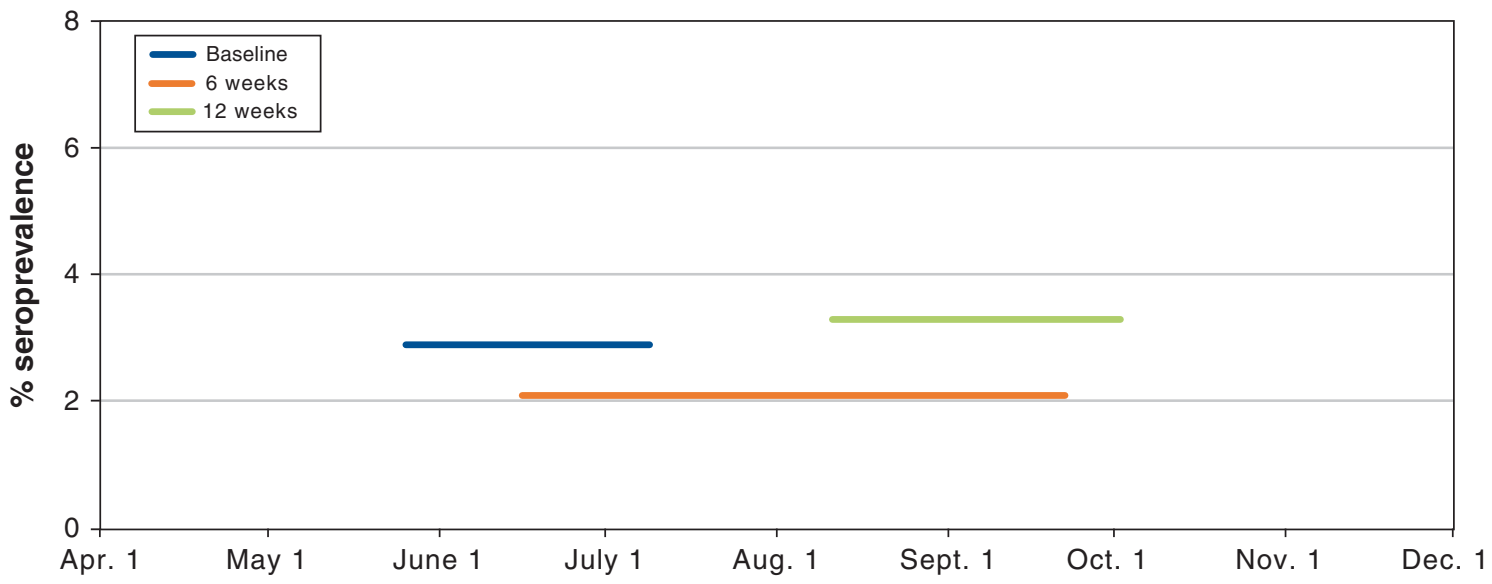

Date, 2020

MHS

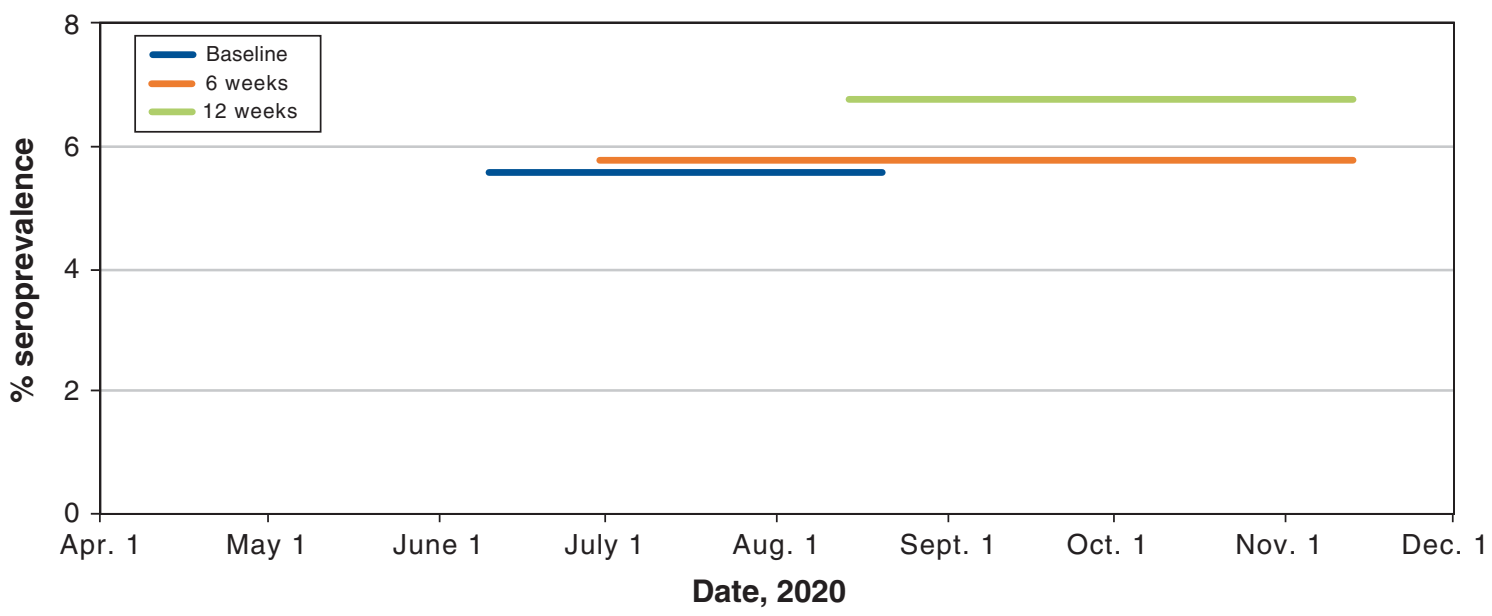

Figure 2: Percentage of participants with positive serology for SARS-CoV-2 by month and by site. Horizontal lines represent the mean percent positivity at enrolment, 6-week or 12-week collection period. Note: LHSC = London Health Sciences Centre, MSH = Markham Stouffville Hospital, SickKids $=$ The Hospital for Sick Children. 


\begin{tabular}{|c|c|c|c|c|}
\hline \multirow[b]{2}{*}{ Characteristic } & \multicolumn{4}{|c|}{ No. $(\%)$ of participants* } \\
\hline & $\begin{array}{c}\text { Total } \\
n=1062\end{array}$ & $\begin{array}{l}\text { SickKids } \\
n=376\end{array}$ & $\begin{array}{l}\text { London Health } \\
\text { Sciences } \\
n=349\end{array}$ & $\begin{array}{c}\text { Markham } \\
\text { Stouffville } \\
n=337\end{array}$ \\
\hline Age, yr, median (IQR) & $40(32-51)$ & $38(31-49)$ & $39(31-52)$ & $42(33-51)$ \\
\hline Sex, female & $834(78.5)$ & $272(72.3)$ & $283(81.1)$ & $279(82.8)$ \\
\hline \multicolumn{5}{|l|}{ Role } \\
\hline Physician & 237 (22.3) & $121(32.2)$ & $66(18.9)$ & $50(14.8)$ \\
\hline Nurse practitioner & $15(1.4)$ & $5(1.3)$ & $3(0.9)$ & $7(2.1)$ \\
\hline Nurse & $446(42.0)$ & $135(35.9)$ & $195(55.9)$ & $116(34.4)$ \\
\hline Allied health worker & $159(15.0)$ & $34(9.0)$ & $47(13.5)$ & $78(23.1)$ \\
\hline Respiratory therapy & $52(4.9)$ & $15(4.0)$ & $20(5.7)$ & $17(5.0)$ \\
\hline Auxiliary health worker & $76(7.2)$ & $41(10.9)$ & $14(4.0)$ & $21(6.2)$ \\
\hline Other† & $115(10.8)$ & $39(10.4)$ & $16(4.6)$ & $60(17.8)$ \\
\hline \multicolumn{5}{|l|}{ Workplace } \\
\hline Emergency department & $306(28.8)$ & $102(27.1)$ & $129(37.0)$ & 75 (22.3) \\
\hline Critical care & $245(23.1)$ & $70(18.6)$ & $125(35.8)$ & $50(14.8)$ \\
\hline Hospital ward & $373(35.1)$ & $121(32.2)$ & $128(36.7)$ & $124(36.8)$ \\
\hline Perioperative services or surgical ward & $157(14.8)$ & $60(16.0)$ & $49(14.0)$ & $48(14.2)$ \\
\hline COVID-19 assessment centre & $37(3.5)$ & $8(2.1)$ & $5(1.4)$ & $24(7.1)$ \\
\hline Otherł & $257(24.2)$ & 99 (26.3) & $51(14.6)$ & $107(31.8)$ \\
\hline No. of individuals in household, median (IQR) & $3(2-4)$ & $3(2-4)$ & $3(2-4)$ & $4(2-4)$ \\
\hline $\begin{array}{l}\text { No. with } \geq 3 \text { individuals in household (including } \\
\text { participant) }\end{array}$ & $602 / 1043(57.7)$ & $178 / 359(49.6)$ & $182 / 349(52.1)$ & $242 / 335(72.2)$ \\
\hline No. with children $(<18 \mathrm{yr})$ in the household & $401(37.8)$ & $122(32.4)$ & $121(34.7)$ & $158(46.9)$ \\
\hline Underlying medical conditions & $386(36.3)$ & $124(33.0)$ & $135(38.7)$ & $127(37.7)$ \\
\hline \multicolumn{5}{|l|}{ Race or ancestry } \\
\hline Inuit, First Nations, Métis & $3(0.3)$ & 0 & $1(0.3)$ & $2(0.6)$ \\
\hline White & $734(69.1)$ & 24364.6 & $296(84.8)$ & $195(57.9)$ \\
\hline Black & $16(1.5)$ & $9(2.4)$ & $3(0.9)$ & $4(1.2)$ \\
\hline Hispanic & $14(1.3)$ & $10(2.7)$ & $3(0.9)$ & $1(0.3)$ \\
\hline Asian & $172(16.2)$ & $52(13.8)$ & $25(7.2)$ & $95(28.2)$ \\
\hline Middle Eastern & $31(2.9)$ & $8(2.1)$ & $12(3.4)$ & $11(3.3)$ \\
\hline Other & $55(5.2)$ & $21(5.6)$ & $7(2.0)$ & $27(8.0)$ \\
\hline Unknown/unspecified & $40(3.8)$ & $33(8.8)$ & $3(0.9)$ & $4(1.2)$ \\
\hline Travel since Jan. 1, 2020 & $402(37.9)$ & 159 (42.3) & $138(39.5)$ & $105(31.2)$ \\
\hline Worked on a unit with a COVID-19 outbreak & $120(11.3)$ & $3(0.8)$ & $93(26.6)$ & $24(7.1)$ \\
\hline Provided direct care to patient with COVID-19 & $439(41.3)$ & $29(7.7)$ & $230(65.9)$ & $180(53.4)$ \\
\hline $\begin{array}{l}\text { Known unprotected occupational exposure with direct } \\
\text { patient care }\end{array}$ & $41 / 439(9.3)$ & $4 / 29(13.8)$ & $24 / 230(10.4)$ & $13 / 180(7.2)$ \\
\hline Known SARS-CoV-2 positive by PCR before enrolment & $40(3.8)$ & $17(4.5)$ & $7(2.0)$ & $16(4.7)$ \\
\hline \multicolumn{5}{|l|}{$\begin{array}{l}\text { Positivity proportion (to SARS-CoV-2 immunoglobulin G } \\
\text { antibodies) }\end{array}$} \\
\hline Overall (at any time point) & $57 / 1062(5.4)$ & $24 / 376(6.4)$ & $12 / 349(3.4)$ & $21 / 337(6.2)$ \\
\hline Baseline (at enrolment) & $48 / 1062(4.5)$ & 19/376 (5.1) & 10/349 (2.9) & $19 / 337(5.6)$ \\
\hline 6 weeks & $53 / 1042(5.1)$ & $22 / 367(6.0)$ & 10/346 (2.9) & $19 / 329(5.8)$ \\
\hline 12 weeks & 48/966 (5.0) & 18/348 (5.2) & $11 / 338(3.3)$ & $19 / 280(6.8)$ \\
\hline
\end{tabular}




\begin{tabular}{|c|c|c|c|}
\hline \multirow[b]{2}{*}{ Serology status } & \multicolumn{2}{|c|}{ Prior PCR status, no. } & \multirow{2}{*}{$\begin{array}{l}\text { Total no. }(\%) \text { of samples positive } \\
\text { and negative at each time point }\end{array}$} \\
\hline & Positive & Negative & \\
\hline \multicolumn{4}{|l|}{ Positive } \\
\hline Baseline & $30 / 40$ & $18 / 1022$ & 48/1062 (4.5) \\
\hline 6 weeks & $30 / 39$ & $21 / 1003$ & $51 / 1042(4.9)$ \\
\hline 12 weeks & $29 / 38$ & $19 / 928$ & 48/966 (6.0) \\
\hline At any point & $31 / 40$ & $26 / 1022$ & $57 / 1062(5.4)$ \\
\hline \multicolumn{4}{|l|}{ Negative } \\
\hline Baseline & $10 / 40$ & $1004 / 1022$ & 1014/1062 (95.5) \\
\hline 6 weeks & 9/39 & $982 / 1003$ & $991 / 1042(95.1)$ \\
\hline 12 weeks & $9 / 38$ & $909 / 928$ & 918/966 (95.0) \\
\hline At any point & $9 / 40$ & $996 / 1022$ & $1005 / 1062$ (94.6) \\
\hline Total & 40 & 1022 & 1062 \\
\hline
\end{tabular}

\section{Antibody responses}

Of the 57 health care workers with positive serology at any time over the course of the study, $48(84.2 \%)$ were positive at baseline testing, and $9(15.8 \%)$ seroconverted during the study. Of the 9 who seroconverted, 1 had a confirmed SARS$\mathrm{CoV}-2$ infection and had baseline testing before 15 days. Of the remaining 8 without previous confirmed infection, 3 were only transiently positive at the 6 -week collection, 1 had more than 1 positive result but at a relatively low antibody index value, and the remaining 4 were positive only on the 12 -week sample with a low antibody index value; none of these participants had confirmed infection over the course of the study.

Figure $3 \mathrm{~A}$ shows the antibody responses in the 26 participants who were antibody positive but had no history of confirmed SARS-CoV-2 infection by PCR testing. Antibody responses of the 31 participants with positive serology and history of previous PCR-confirmed infection are shown in Figure 3B (by month) and Figure 3C (days since positive PCR test).

\section{Predictors of seropositivity}

A comparison of clinical and other possible exposures by detectable antibody status is summarized in Table 3 (additional factors are described in Appendix 2 available at www. cmajopen.ca/content/9/4/E929/suppl/DC1, Supplementary Table 1). Seroprevalence was higher among those who had a known unprotected exposure to a patient with COVID-19 $(29.6 \%$ v. $8.0 \%, p<0.001)$, those who had been contacted by public health because of a nonhospital exposure $(15.8 \% \mathrm{v}$. $5.5 \%, p=0.003)$ and those with confirmed infection before enrolment $(54.4 \%$ v. $0.9 \%, p<0.001)$. Working on a unit with a COVID-19 outbreak was not associated with higher seroprevalence $(8.8 \%$ v. $11.5 \%, p=0.7)$.

In the multivariable model (Table 4), presence of symptomatic contacts in the household was the strongest predic- tor of seropositivity (adjusted odds ratio [OR] 7.15, 95\% CI 5.42-9.41). When health care workers with known infection at baseline were removed, several other predictors were identified. Presence of symptomatic contacts in the household remained a strong predictor (adjusted OR 7.22, 95\% CI 3.65-14.3). Younger age by year (adjusted OR 0.94, 95\% CI 0.91-0.98) and non-White race (adjusted OR 2.85, 95\% CI 1.36-5.98) were also found to be significant. Providing direct care to patients with COVID-19 was found to be associated with a lower odds of infection (adjusted OR 0.50, 95\% CI 0.36-0.70).

\section{Symptom history}

A total of $47.9 \%(n=23)$ of health care workers with positive serology at baseline reported a history of symptomatic illness (52.1\% asymptomatic). The most reported symptoms included cough $(n=17,35.4 \%)$ and fatigue $(n=17,35.4 \%)$ (Appendix 2, Supplementary Table 2). Those with symptoms documented at least 2 symptoms $(n=22)$, with 1 health care worker reporting isolated anosmia.

\section{Interpretation}

Among the health care workers sampled across several Ontario hospital sites, including a community hospital, tertiary care pediatric hospital and a combined adult-pediatric academic health centre, seroprevalence of SARS-CoV-2 antibodies was $5.4 \%$. The prevalence was even lower, at $2.5 \%$, when we took into account recruitment bias of prior infection before enrolment. Among health care workers, the main risk factors identified for seroprevalence were outside of the hospital (household or community exposure), unless the worker had a known unprotected health care exposure.

Our finding of $2 \%-5 \%$ prevalence of seropositivity depending on prior infection is consistent with most findings of other 
A

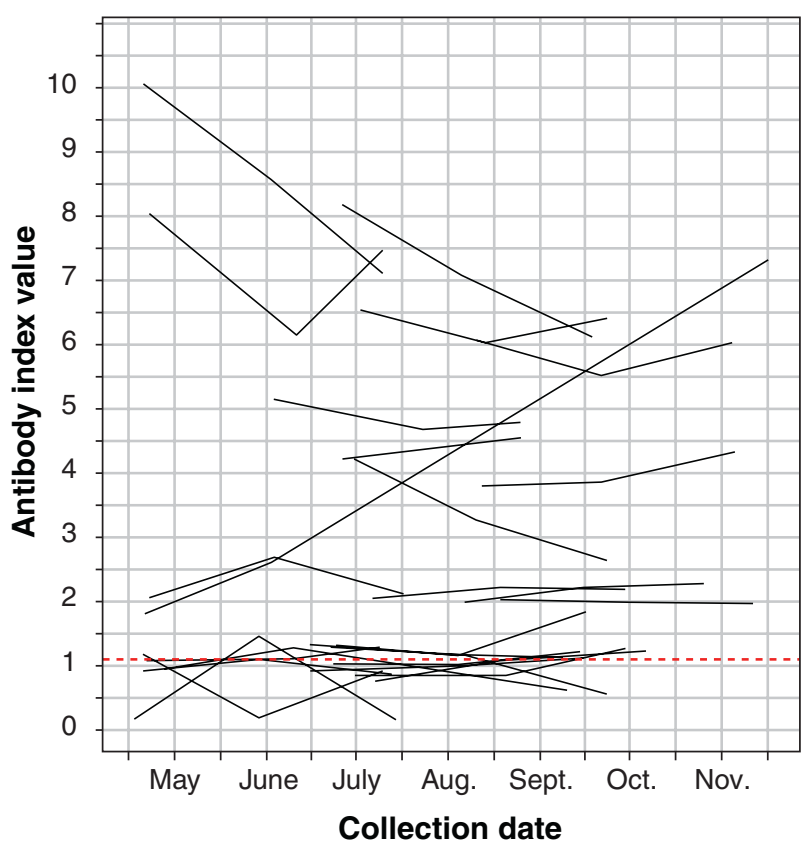

B

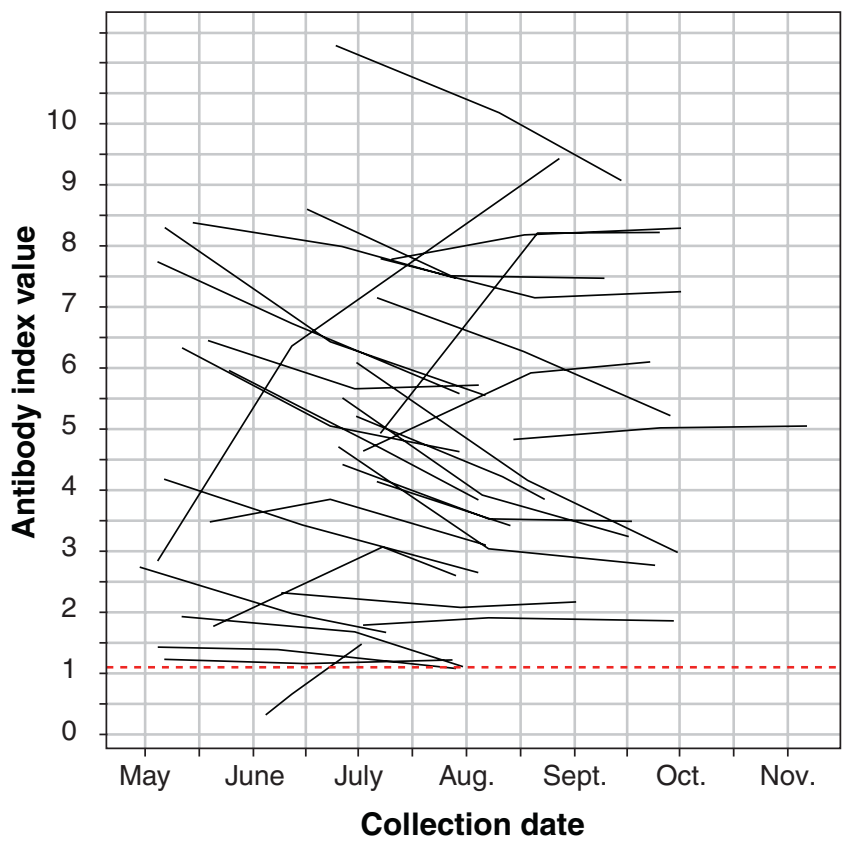

C

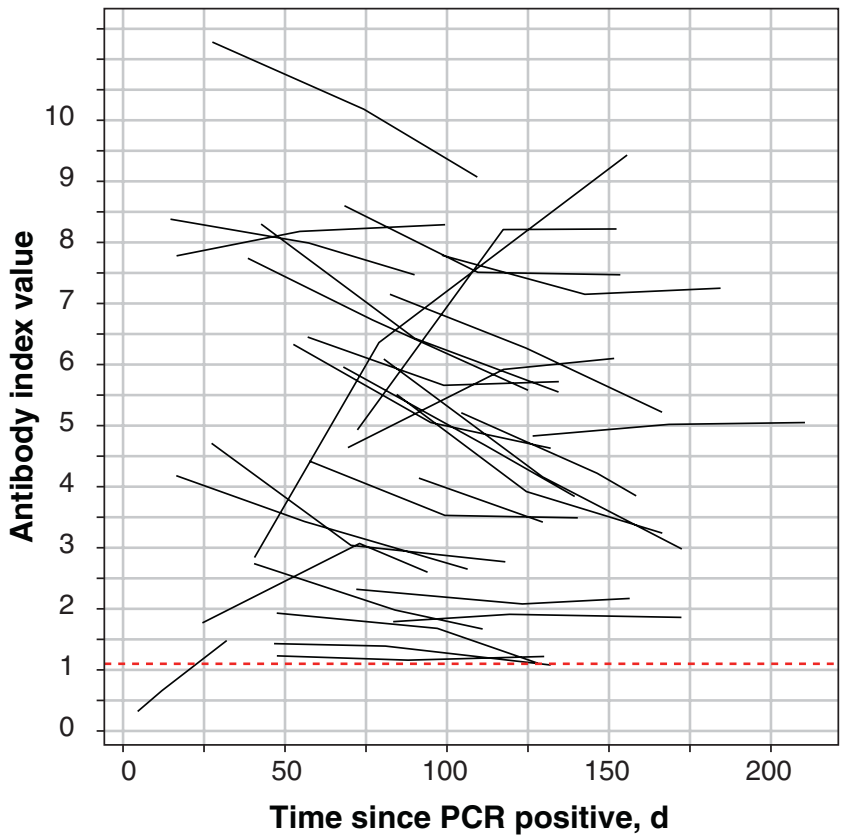

Figure 3: (A) Antibody responses of the 26 participants who had no history of confirmed SARS-CoV-2 infection and tested positive for SARSCoV-2 antibodies at any time point during the study. Points above the dashed red line represent a positive antibody result. (B) Antibody responses of the 31 participants who had confirmed SARS-CoV-2 infection by polymerase chain reaction (PCR) testing and tested positive for SARS-CoV-2 antibodies at any time point during the study by collection time. Points above the dashed red line represent a positive antibody result. (C) Antibody responses of the 31 participants who had confirmed SARS-CoV-2 infection by molecular testing and tested positive for SARS-CoV-2 antibodies at any time point during the study expressed as a time from their positive PCR result. Points above the dashed red line represent a positive antibody result. 


\begin{tabular}{|c|c|c|c|}
\hline \multirow[b]{2}{*}{ Variable } & \multicolumn{2}{|c|}{ No. (\%) of participants } & \multirow[b]{2}{*}{$p$ value } \\
\hline & $\begin{array}{c}\text { SARS-CoV-2 } \\
\text { serology positive } \\
\quad n=57^{*}\end{array}$ & $\begin{array}{c}\text { SARS-CoV-2 } \\
\text { serology negative } \\
\quad n=1005^{\star}\end{array}$ & \\
\hline Symptomatic contacts in the household & 7/53 (13.2) & 25/971 (2.6) & $<0.001$ \\
\hline Provided direct care to patients with COVID-19 & $27(47.4)$ & $412 / 995(41.4)$ & 0.5 \\
\hline $\begin{array}{l}\text { Unprotected occupational exposure to a patient } \\
\text { with COVID-19† }\end{array}$ & $8 / 27(29.6)$ & $33 / 411(8.0)$ & $<0.001$ \\
\hline Worked on a unit with a COVID-19 outbreak & $5(8.8)$ & $115 / 996(11.5)$ & 0.7 \\
\hline Contacted by public health to indicate exposure & $9(15.8)$ & $54 / 985(5.5)$ & 0.003 \\
\hline Known positive PCR test at baseline & $31(54.4)$ & $9(0.9)$ & $<0.001$ \\
\hline \multicolumn{4}{|c|}{$\begin{array}{l}\text { Note: PCR = polymerase chain reaction. } \\
\text { *The denominator of each outcome variable is the total in the column heading unless stated otherwise. } \\
\text { tOnly those health care workers who indicated they had direct patient contact were asked this question. }\end{array}$} \\
\hline
\end{tabular}

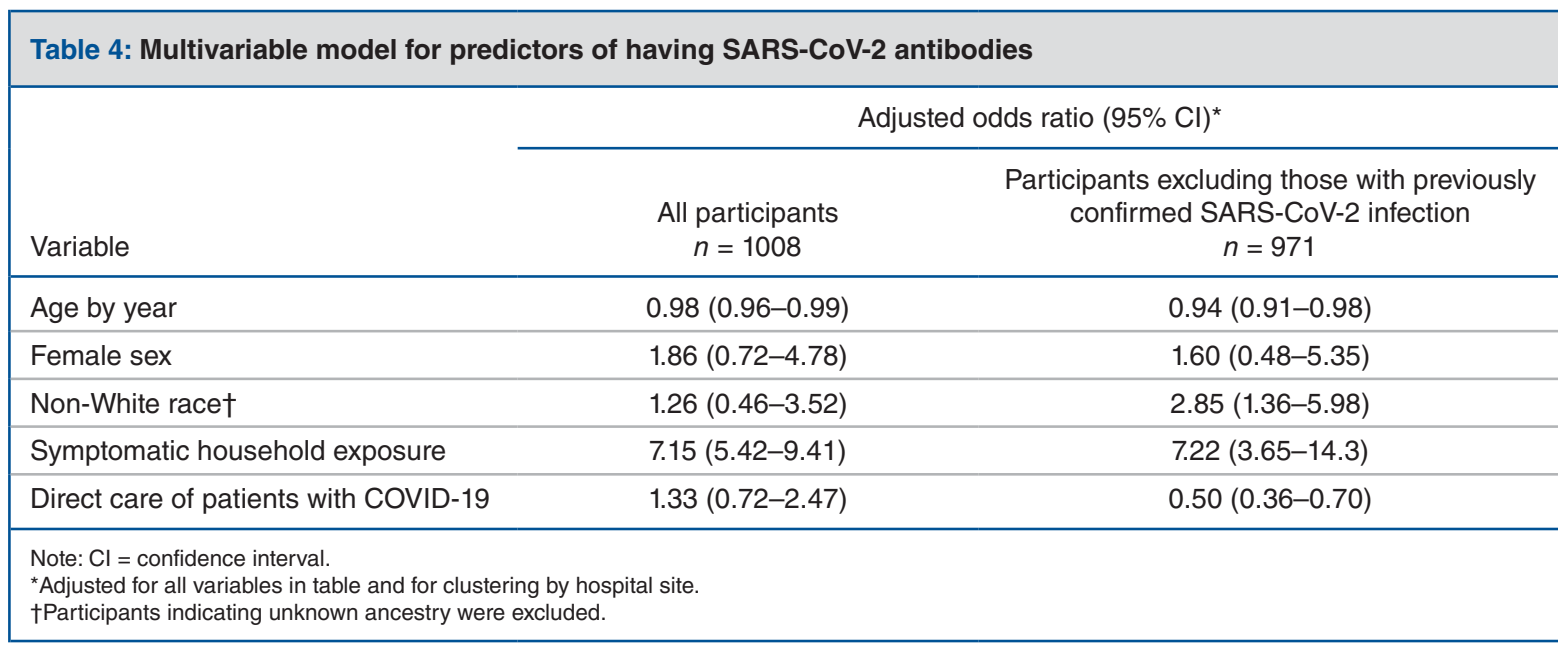

seroprevalence studies involving health care workers, in which seroprevalence ranged from $0 \%$ to $44 \%$, depending on the jurisdiction. ${ }^{8,9111,12,21-33}$ Since the start of the pandemic, given the experience with severe acute respiratory syndrome $(\mathrm{SARS})^{34-36}$ and studies of SARS-CoV-2 showing environmental contamination ${ }^{37}$ and occasionally (though not consistently) presence in air samples, there was a concern of higher prevalence of infection among health care workers. ${ }^{38,39}$ Not surprisingly, we found higher seroprevalence among health care workers from jurisdictions with higher community rates. Overall, seroprevalence in the 2 hospitals from the Greater Toronto Area (where community rates and seroprevalence were higher $\left.{ }^{16,40}\right)$ was $6.4 \%$ (2.5\% excluding known positives) and $6.2 \%$ (3.1\% excluding known positives), whereas in southwestern Ontario (a community where incidence and seroprevalence were lower) it was $3.4 \%$ (2.0\% excluding known positives).

In addition to variation in COVID-19 burden by region, $, 12,28,31$ studies showing higher seroprevalence among health care workers attributed these estimates to availability of $\mathrm{PPE}^{27,32}$ and delayed implementation of public health measures in the hospital (i.e., universal masking). ${ }^{28,29}$ Shortages of PPE and episodes of lacking facial coverings while caring for patients with COVID-19 (defined as lack of surgical mask, N95 respirator or powered air purifying respirator [PAPR]), were associated with seropositivity in a multicentre US-based serosurvey. ${ }^{24}$ This is in line with our finding of a higher odds of infection among health care workers who had unprotected exposures with patients with COVID-19. Across our hospitals, as across Canada, medical masks are used as part of droplet and contact precautions for routine care of patients, with N95 respirator or PAPRs recommended for use only in aerosol-generating medical procedures. ${ }^{17}$ This approach differs from that of the United States, where an N95 respirator or PAPR is recommended for all encounters with patients with COVID-19, while acknowledging that medical masks are an acceptable alternative. ${ }^{41}$ Although further studies are needed, our results suggest a lack of substantially different seroprevalence in our health care workers compared with either rates in the local communities or other health care workers involved in seroprevalence studies in other countries. 
This is reassuring that our current infection prevention and control practices appear to be effective.

We found that exposure to a symptomatic household member was the strongest predictor of positive serology, and providing direct care to patients with COVID-19 or working on a unit with a COVID-19 outbreak was not a significant predictor. Evidence supporting household exposure as potentially contributing more to infection risk than the health care environment has been previously described. Wilkins and colleagues found that exposure outside of the hospital was strongly associated with seropositivity in a large seroprevalence study involving health care workers in Chicago, ${ }^{13}$ and Steensels and colleagues found that having a household contact with suspected COVID-19 was strongly associated with seropositivity. ${ }^{11}$ Additionally, younger age and non-White race were significant predictors of seropositivity, a finding described in other studies ${ }^{42,43}$ and consistent with community risk factors. ${ }^{44}$

Only about half of the health care workers with antibodies in our study reported signs or symptoms of COVID-19. Similar prevalence findings among asymptomatic or paucisymptomatic health care workers with positive serology were documented in other studies..$^{923-28,45}$ This highlights the need for a low threshold for testing among health care workers as well as ensuring that health and safety measures are followed consistently in hospitals and the community.

The longitudinal collection of samples allowed for the evaluation of the durability of the antibody response. Present evidence suggests that measurable antibody responses may decrease over time. ${ }^{46-48}$ This decline has been observed in assays using the SARS-CoV-2 S protein, including the one used for this study. ${ }^{49}$ It was surprising that a decline in antibody levels that resulted in a change of serostatus from positive to negative was rare, occurring in only 3 health care workers who had positive baseline serology, in contrast to the significant decline of more than $50 \%$ seen over a 60 -day period among health care workers in a study by Patel and colleagues. ${ }^{46}$

\section{Limitations}

Limitations of this study include the convenience sampling of health care workers and modest sample size. Owing to logistical difficulties in bringing on study sites midpandemic, only 3 sites were included in this analysis, which focuses on the first wave. In addition, given the passive and broad nature of recruitment, it is difficult to know the exact number of health care workers notified about the study at each site to obtain an accurate recruitment rate. Ongoing recruitment at additional hospital sites has also focused on increasing the number of high-risk workers.

Our study had low power to detect differences between seropositive and seronegative groups. Furthermore, as commonly seen in studies assessing seroprevalence, there may have been a recruitment bias toward health care workers who suspected previous infection and were interested in their antibody response (e.g., history of undiagnosed respiratory symptoms or previous confirmed infection). In terms of the riskfactor assessment, questionnaires were self-completed.
However, antibody testing was batched, and questionnaires were completed before results were available, so the results should not have biased the responses.

The serologic response to SARS-CoV-2 can cross-react with antibodies after infections with SARS, Middle East respiratory syndrome coronavirus (MERS-CoV) and other seasonal coronaviruses in circulation. ${ }^{50}$ Two individuals with selfreported previous infection with SARS or MERS-CoV were tested, with 1 being seropositive. Although we did not perform orthogonal testing with an alternative target antigen, we followed patient status over time as a mitigation strategy, with $87 \%$ of participants with positive testing remaining positive on more than 1 blood collection. False-negative results may have occurred because the assay may have failed to detect a measurable antibody response from a limitation in its sensitivity. ${ }^{51-53}$ False-negative results may also occur if a participant did not mount a robust antibody response or if the antibody response waned before recruitment. ${ }^{54,55}$ Additionally, the assay used was not quantitative, and instead signal to cut-off ratios were used as a surrogate for antibody titres.

\section{Conclusion}

We found that health care workers with community risk factors such as household or community exposure were more likely to be seropositive with SARS-CoV-2 antibodies, and direct care of patients with COVID-19 was not associated with increased seropositivity. Our results highlight the importance of public health measures both inside and outside of the hospital.

\section{References}

1. Kampf G, Bruggemann Y, Kaba HEJ, et al. Potential sources, modes of transmission and effectiveness of prevention measures against SARS-CoV-2. 7 Hosp Infect 2020;106:678-97.

2. Reynolds MG, Anh BH, Thu VH, et al. Factors associated with nosocomial SARS-CoV transmission among healthcare workers in Hanoi, Vietnam, 2003. BMC Public Health 2006;6:207.

3. Schwartz J, King CC, Yen MY. Protecting healthcare workers during the coronavirus disease 2019 (COVID-19) outbreak: lessons from Taiwan's severe acute respiratory syndrome response. Clin Infect Dis 2020;71:858-60.

4. Havers FP, Reed C, Lim T, et al. Seroprevalence of antibodies to SARSCoV-2 in 10 sites in the United States, March 23-May 12, 2020. FAMA Intern Med 2020 July 21. doi: 10.1001/jamainternmed.2020.4130. [Epub ahead of print].

5. Loeffelholz MJ, Tang YW. Laboratory diagnosis of emerging human coronavirus infections - the state of the art. Emerg Microbes Infect 2020;9:747-56.

6. Xiang F, Wang X, He X, et al. Antibody detection and dynamic characteristics in patients with coronavirus disease 2019. Clin Infect Dis 2020;71:1930-4.

7. Zhao J, Yuan Q, Wang H, et al. Antibody responses to SARS-CoV-2 in patients with novel coronavirus disease 2019. Clin Infect Dis 2020;71:2027-34.

8. Iversen K, Bundgaard H, Hasselbalch RB, et al. Risk of COVID-19 in health-care workers in Denmark: an observational cohort study. Lancet Infect Dis 2020;20:1401-8.

9. Houlihan CF, Vora N, Byrne T, et al. Pandemic peak SARS-CoV-2 infection and seroconversion rates in London frontline health-care workers. Lancet 2020;396:e6-7.

10. Lai X, Wang M, Qin C, et al. Coronavirus disease 2019 (COVID-2019) infection among health care workers and implications for prevention measures in a tertiary hospital in Wuhan, China. 7AMA Netw Open 2020;3: e209666.

11. Steensels D, Oris E, Coninx L, et al. Hospital-wide SARS-CoV-2 antibody screening in 3056 staff in a tertiary center in Belgium. FAMA 2020;324: 195-7.

12. Moscola J, Sembajwe G, Jarrett M, et al. Prevalence of SARS-CoV-2 antibodies in health care personnel in the New York City area. $7 A M A 2020$; 324:893-5.

13. Wilkins JT, Gray EL, Wallia A, et al. Seroprevalence and correlates of SARSCoV-2 antibodies in health care workers in Chicago. Open Forum Infect Dis 2020;8:ofaa582. doi:10.1093/ofid/ofaa582. 
14. Nguyen LH, Drew DA, Graham MS, et al. Risk of COVID-19 among frontline health-care workers and the general community: a prospective cohort study. Lancet Public Health 2020;5:e475-e483.

15. Lidstrom AK, Sund F, Albinsson B, et al. Work at inpatient care units is associated with an increased risk of SARS-CoV-2 infection; a cross-sectional study of 8679 healthcare workers in Sweden. Ups 7 Med Sci 2020;125:305-10.

16. Epidemiologic summary: COVID-19 in Ontario - January 15, 2020 to December 20, 2020. Toronto: Ontario Agency for Health Protection and Promotion (Public Health Ontario); 2020. Available: https://www.publichealthontario.ca/-/ media/documents/ncov/epi/2020/covid-19-daily-epi-summary-report.pdf?la=en (accessed 2021 Apr. 30).

17. IPAC recommendations for use of personal protective equipment for care of individuals with suspect or confirmed COVID-19. Toronto: Ontario Agency for Health Protection and Promotion (Public Health Ontario); 2020. Available: https://www.publichealthontario.ca/-/media/documents/ncov/updated-ipac -measures-covid-19.pdf?la=en (accessed 2020 Dec.19).

18. Protocol for assessment of potential risk factors for 2019-novel coronavirus (COVID-19) infection among health care workers in a health care setting. Geneva: World Health Organization; 2020. Available: https://who.int/ publications-detail/protocol-for-assessment-of-potential-risk-factors-for-2019 -novel-coronavirus-(2019-ncov)-infection-among-health-care-workers-in-a -health-care-setting (accessed 2020 Dec. 19).

19. SARS-CoV-2 ELISA test systems from EUROIMMUN. Luebeck (Germany): EUROIMMUN Medizinische Labordiagnostika AG. Available: https://www. coronavirus-diagnostics.com/antibody-detection-tests-for-covid-19.html (accessed 2020 Dec. 19).

20. Theel ES, Harring J, Hilgart H, et al. Performance characteristics of four high-throughput immunoassays for detection of IgG antibodies against SARS-CoV-2.7 Clin Microbiol 2020;58:e01243-20.

21. Jeremias A, Nguyen J, Levine J, et al. Prevalence of SARS-CoV-2 infection among health care workers in a tertiary community hospital. 7 AMA Intern Med 2020;180:1707-9.

22. Barrett ES, Horton DB, Roy J, et al. Prevalence of SARS-CoV-2 infection in previously undiagnosed health care workers in New Jersey, at the onset of the U.S. COVID-19 pandemic. BMC Infect Dis 2020;20:853.

23. Stubblefield WB, Talbot HK, Feldstein L, et al. Seroprevalence of SARSCoV-2 among frontline healthcare personnel during the first month of caring for COVID-19 patients — Nashville, Tennessee. Clin Infect Dis 2020;72:1645-48.

24. Self WH, Tenforde MW, Stubblefield WB, et al. Seroprevalence of SARSCoV-2 among frontline health care personnel in a multistate hospital network - 13 academic medical centers, April-June 2020. MMWR Morb Mortal Wkly Rep 2020;69:1221-6.

25. Korth J, Wilde B, Dolff S, et al. SARS-CoV-2-specific antibody detection in healthcare workers in Germany with direct contact to COVID-19 patients. Z Clin Virol 2020;128:104437.

26. Godbout EJ, Pryor R, Harmon M, et al. COVID-19 seroprevalence among healthcare workers in a low prevalence region. Infect Control Hosp Epidemiol 2020 Dec. 14 [Epub ahead of print]. doi: 10.1017/ice.2020.1374.

27. Mansour M, Leven E, Muellers K, et al. Prevalence of SARS-CoV-2 antibodies among healthcare workers at a tertiary academic hospital in New York City. 7 Gen Intern Med 2020;35:2485-6.

28. Grant JJ, Wilmore SMS, McCann NS, et al. Seroprevalence of SARS-CoV-2 antibodies in healthcare workers at a London NHS Trust. Infect Control Hosp Epidemiol 2021;42:212-4.

29. Rudberg AS, Havervall S, Manberg A, et al. SARS-CoV-2 exposure, symptoms and seroprevalence in healthcare workers in Sweden. Nat Commun 2020;11:5064.

30. Eyre DW, Lumley SF, O'Donnell D, et al. Differential occupational risks to healthcare workers from SARS-CoV-2 observed during a prospective observational study. eLife 2020;9:e60675. doi: 10.7554/eLife.60675.

31. Galan MI, Velasco M, Casas ML, et al. Hospital-wide SARS-CoV-2 seroprevalence in health care workers in a Spanish teaching hospital. Enferm Infecc Microbiol Clin (Engl Ed) 2020 Dec. 18 [Epub ahead of print]. doi:10.1016/j. eimc.2020.11.015.

32. Chen $\mathrm{Y}$, Tong $\mathrm{X}$, Wang $\mathrm{J}$, et al. High SARS-CoV-2 antibody prevalence among healthcare workers exposed to COVID-19 patients. $f$ Infect 2020; 81:420-6.

33. Insua C, Stedile G, Figueroa V, et al. Seroprevalence of SARS-CoV-2 antibodies among physicians from a children's hospital. Arch Argent Pediatr 2020;118:381-5.

34. Varia M, Wilson S, Sarwal S, et al. Investigation of a nosocomial outbreak of severe acute respiratory syndrome (SARS) in Toronto, Canada. CMA7 2003; 169:285-92.

35. Masur H, Emanuel E, Lane HC. Severe acute respiratory syndrome: providing care in the face of uncertainty. $7 A M A$ 2003;289:2861-3.

36. Fowler RA, Guest CB, Lapinsky SE, et al. Transmission of severe acute respiratory syndrome during intubation and mechanical ventilation. Am 7 Respir Crit Care Med 2004;169:1198-202.

37. Ye G, Lin H, Chen S, et al. Environmental contamination of SARS-CoV-2 in healthcare premises. 7 Infect 2020;81:e1-5.

38. Guo ZD, Wang ZY, Zhang SF, et al. Aerosol and surface distribution of severe acute respiratory syndrome coronavirus 2 in hospital wards, Wuhan, China, 2020. Emerg Infect Dis 2020;26:1583-91.
39. Li YH, Fan YZ, Jiang L, et al. Aerosol and environmental surface monitoring for SARS-CoV-2 RNA in a designated hospital for severe COVID-19 patients. Epidemiol Infect 2020;148:e154.

40. COVID-19 seroprevalence in Ontario: March 27, 2020 to June 30, 2020 Toronto: Ontario Agency for Health Protection and Promotion (Public Health Ontario); 2020. Available: https://www.publichealthontario.ca/-/ media/documents/ncov/epi/2020/07/covid-19-epi-seroprevalence-in-ontario. pdf?la=en (accessed 2021 Apr. 30).

41. Interim infection prevention and control recommendations for healthcare personnel during the coronavirus disease 2019 (COVID-19) pandemic. Atlanta: Centers for Disease Control and Prevention; updated 2020 Dec. 14. Available: https:// www.cdc.gov/coronavirus/2019-ncov/hcp/infection-control-recommendations. html (accessed 2020 Dec. 19).

42. Rosser JI, Roltgen K, Dymock M, et al. SARS-CoV-2 seroprevalence in healthcare personnel in Northern California early in the COVID-19 pandemic. Infect Control Hosp Epidemiol. 2020 Dec. 9 [Epub ahead of print]. doi: 10.1017/ice.2020.1358.

43. Ebinger JE, Botwin GJ, Albert CM, et al. Seroprevalence of antibodies to SARS-CoV-2 in healthcare workers: a cross-sectional study. BMF Open 2021;11(2):e043584. doi:10.1136/bmjopen-2020-043584

44. COVID-19 in Ontario - a focus on diversity: January 15, 2020 to May 14, 2020 Toronto: Ontario Agency for Health Protection and Promotion (Public Health Ontario); 2020. Available: https://www.publichealthontario.ca/-/ media/documents/ncov/epi/2020/06/covid-19-epi-diversity.pdf?la=en (accessed 2020 Dec. 19).

45. Garcia-Basteiro AL, Moncunill G, Tortajada M, et al. Seroprevalence of antibodies against SARS-CoV-2 among health care workers in a large Spanish reference hospital. Nat Commun 2020;11:3500.

46. Patel MM, Thornburg NJ, Stubblefield WB, et al. Change in antibodies to SARS-CoV-2 Over 60 days among health care personnel in Nashville, Tennessee. 7AMA 2020;324:1781-2.

47. Seow J, Graham C, Merrick B, et al. Longitudinal observation and decline of neutralizing antibody responses in the three months following SARS-CoV-2 infection in humans. Nat Microbiol 2020;5:1598-607.

48. Roltgen K, Powell AE, Wirz OF, et al. Defining the features and duration of antibody responses to SARS-CoV-2 infection associated with disease severity and outcome. Sci Immunol 2020;5:eabe0240.

49. Choe PG, Kang CK, Suh HJ, et al. Waning antibody responses in asymptomatic and symptomatic SARS-CoV-2 infection. Emerg Infect Dis 2021; 27:327-9.

50. Lisboa Bastos M, Tavaziva G, Abidi SK, et al. Diagnostic accuracy of serological tests for covid-19: systematic review and meta-analysis. BMF 2020;370:m2516.

51. Van Elslande J, Houben E, Depypere M, et al. Diagnostic performance of seven rapid IgG/IgM antibody tests and the Euroimmun IgA/IgG ELISA in COVID-19 patients. Clin Microbiol Infect 2020;26:1082-7.

52. Manthei DM, Whalen JF, Schroeder LF, et al. Differences in performance characteristics among four high-throughput assays for the detection of antibodies against SARS-CoV-2 using a common set of patient samples. Am $\mathcal{F}$ Clin Pathol 2021;155:267-79.

53. Beavis KG, Matushek SM, Abeleda APF, et al. Evaluation of the EUROIMMUN anti-SARS-CoV-2 ELISA assay for detection of IgA and IgG antibodies. 7 Clin Virol 2020;129:104468.

54. Plebani M. Antibody responses in mild COVID-19 hospital staff. EBioMedicine 2020;59:102940.

55. Ibarrondo FJ, Fulcher JA, Goodman-Meza D, et al. Rapid decay of antiSARS-CoV-2 antibodies in persons with mild COVID-19. $N$ Engl 7 Med 2020;383:1085-7.

Competing interests: Shelly Bolotin has received grants for COVID-19 work, unrelated to this manuscript, from the Canadian Institutes of Health Research (CIHR), the Public Health Agency of Canada (PHAC), the COVID-19 Immunity Task Force (CITF) and the Canadian Immunity Research Network; has participated on the executive management committee for the MOSAIC COVID-19 vaccine clinical trial (CITF funded); and is a member of the PHAC Vaccine Surveillance Reference Group and the Canadian Immunization Research Network Management Committee. Michael Silverman reports an unrestricted grant paid to Western University from the Academic Medical Organization of Southwestern Ontario. Allison McGeer reports research grants to her institution from Sanofi Pasteur, Pfizer, Merck, CIHR, CITF, University of Toronto and Appili Therapeutics; funding from AstraZeneca for the preparation and delivery of webinars about vaccination; and participation on a data safety monitoring board or advisory board regarding COVID19 therapy and vaccines for AstraZeneca, Janssen, Moderna, Pfizer, GlaxoSmithKline, Sanofi Pasteur, Merck, Medicago and Novavax. Upton Allen reports an honorarium provided to SickKids from the St. Jude Pediatric Infectious Diseases course. Jerome Leis reports a grant payment from the University of Toronto COVID-19 Action Initiative to his 
organization, Sunnybrook Health Sciences Centre, as a participant site; and payment for expert testimony regarding COVID-19 infection prevention and control management for the Ontario Hospital Association and the Ontario Ministry of the Attorney General. Jeffrey Pernica reports grant funding from bioMérieux to his institution for a clinical trial and from MedImmune to his institution for a prospective cohort study; and payment for an educational event from the Canadian Paediatric Review. No other competing interests were declared.

Affiliations: Division of Infectious Diseases (Science, Allen, Kazmi, Liu, Sumaida), Department of Paediatrics, The Hospital for Sick Children; Public Health Ontario (Science, Bolotin, Schwartz); Dalla Lana School of Public Health (Bolotin, Ariyarajah), University of Toronto, Toronto, Ont.; Division of Infectious Diseases (Silverman), Department of Medicine and Department of Epidemiology and Biostatistics (Silverman), Western University, London, Ont.; Division of Infectious Diseases (Nadarajah), Markham Stouffville Hospital, Markham, Ont.; Department of Medicine (Nadarajah, Parekh, Leis), University of Toronto; SickKids Research Institute (Maguire, Schneiderman), The Hospital for Sick Children; Division of Nephrology (Parekh), Department of Pediatrics, The Hospital for Sick Children and University of Toronto; Department of Laboratory Medicine and Pathobiology (McGeer, Katz, Campigotto), University of Toronto; Lunenfeld-Tanenbaum Research Institute (McGeer), Sinai Health System, Toronto; Division of Infectious Diseases (Schwartz, Downing), Department of Medicine, Unity Health Toronto; Occupational Health and Safety (Alexander), The Hospital for Sick Children, Toronto, Ont.; Division of Infection Prevention and Control (Castellani), Sault Area Hospital, Sudbury, Ont.; Departments of Paediatrics and Molecular Genetics (Cohn), University of Toronto; Department of Paediatrics (Cohn), The Hospital for Sick Children; Division of Infection Prevention and Control (Katz), North York General Hospital; Division of Infectious Diseases (Leis), Sunnybrook Health Sciences Centre, Toronto, Ont.; Department of Pediatrics (Pernica), McMaster University, Hamilton, Ont.; Division of Microbiology (Campigotto), Department of Paediatric Laboratory Medicine, The Hospital for Sick Children, Toronto, Ont.

Contributors: Shelly Bolotin, Aaron Campigotto, Rulan Parekh, Michelle Science and Michael Silverman conceived and designed the work; contributed to the acquisition, analysis and interpretation of data; and drafted the manuscript. Jeya Nadarajah contributed to the acquisition, analysis and interpretation of data. Bryan Maguire performed the statistical analysis. Allison McGeer, Kevin Schwartz, Laura Alexander, Upton Allen, Archchun Ariyarajah, Lucas Castellani, Ronald Cohn, Mark Downing, Kevin Katz, Kescha Kazmi, Jerome Leis, Derek Liu, Jeffrey Pernica, Jane Schneiderman and Maya Sumaida contributed to the interpretation of the data for the work. All of the authors revised the manuscript critically for important intellectual content, gave final approval of the version to be published and agreed to be accountable for all aspects of the work.

Funding: The Hospital for Sick Children Foundation, University of Toronto COVID-19 Action Initiative, Ontario COVID-19 Rapid Research Fund and Academic Medical Organization of Southwestern Ontario provided funding.

Content licence: This is an Open Access article distributed in accordance with the terms of the Creative Commons Attribution (CC BY-NC-ND 4.0) licence, which permits use, distribution and reproduction in any medium, provided that the original publication is properly cited, the use is noncommercial (i.e., research or educational use), and no modifications or adaptations are made. See: https://creativecommons.org/licenses/ by-nc-nd/4.0/

Data sharing: The data set from this study are held securely in coded form at The Hospital for Sick Children. As subsequent analyses will be conducted after the completion of the larger study, an anonymized subset of data may be made available beginning 3 months and ending 5 years after publication to researchers who provide a methodologically sound proposal. Proposals should be directed to Michelle Science (michelle.science@sickkids.ca) or Aaron Campigotto (aaron.campigotto@sickkids.ca). Research ethics board approval and a data access agreement will need to be signed to gain access to the data.

Acknowledgements: The authors extend their appreciation to Supriya Parikh for her support with project and data management, and James Wright for his support with the manuscript figures.

Supplemental information: For reviewer comments and the original submission of this manuscript, please see www.cmajopen.ca/content/9/4/ E929/suppl/DC1. 